\title{
DISCURSO POLÍTICO SOBRE LA LEY DE EXTRANJERÍA EN LA PRENSA ESPAÑOLA
}

\section{The political discourse around the law on foreigners in the Spanish press}

\section{Antonio Prieto-Andrés}

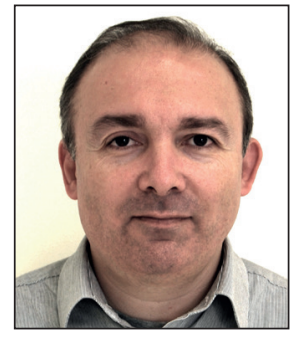

Antonio Prieto-Andrés es docente e investigador en la Facultad de Comunicación y Ciencias Sociales de la Universidad San Jorge de Zaragoza. Doctor en Comunicación, su docencia está vinculada a los derechos humanos, el derecho de la comunicación, la legislación del videojuego y la deontología periodística. Pertenece al grupo de investigación Migraciones, interculturalidad y desarrollo humano, desde el que hace sus aportaciones en relación con los aspectos jurídicos y sociales de la inmigración, así como su tratamiento en la prensa desde una perspectiva de derechos humanos. http://orcid.org/0000-0001-5214-1794

Universidad San Jorge, Facultad de Comunicación y Ciencias Sociales Campus Universitario Villanueva de Gállego Autovía A-23 Zaragoza-Huesca, Km. 299. 50830 Villanueva de Gállego (Zaragoza), España aprieto@usj.es

\section{Resumen}

La inmigración y cómo regularla son temas clave en la sociedad actual y, como tales, son objeto del discurso político que pretende mostrar los argumentos de los diferentes actores sociales, especialmente de los partidos políticos. La prensa es uno de los principales mecanismos que emplean estos actores sociales para mostrar dichos discursos. De ahí que en este trabajo se analice cómo seis diarios españoles presentan los discursos políticos en torno a la Ley de extranjería, como principal instrumento jurídico-político para gestionar la inmigración, a lo largo del período 2003-2016, así como la posición de los partidos políticos que aparecen como fuente principal. Para lograrlo, se ha trabajado con un modelo de Zapata-Barrero, que ha categorizado los discursos políticos sobre la inmigración en dos tipos: discursos pro-activos y discursos re-activos, según muestren su preferencia por gestionar la multiculturalidad que conlleva la inmigración como un reto o como un problema, respectivamente.

\section{Palabras clave}

Inmigración; Multiculturalidad; Ley de extranjería; Discurso político; Partidos políticos; Medios de comunicación; Prensa; Diarios; España.

\begin{abstract}
Immigration and its regulation are key issues in society and, as such, are subject to political discourses that show the arguments of the different social actors, especially political parties. Press is one of the main mechanisms used by these social actors to show such discourses. Hence, this paper analyzes how six Spanish newspapers presented the political discourses on the Foreigners law, as the main legal-political instrument to manage immigration, from 2003 to 2016, as well as the position of the political parties that were the main sources of discourse. To achieve this, we worked with the Zapata-Barrero model, which categorizes political discourses on immigration into two types: pro-active discourses and re-active discourses, which shows their preference for managing multiculturalism as a challenge or a problem, respectively.
\end{abstract}

\section{Keywords}

Immigration; Multiculturalism; Spanish Law on foreigners; Political discourse; Political parties; Media; Press; Newspapers; Spain.

Prieto-Andrés, Antonio (2017). "Discurso político sobre la Ley de extranjería en la prensa española". El profesional de la información, v. 26, n. 4, pp. 695-704. 


\section{Introducción}

La inmigración es un asunto muy relevante en la sociedad actual, por lo que es objeto de múltiples acciones políticas con el fin de gestionarla. El principal instrumento jurídico-político de regulación en España es la Ley Orgánica 4/2000, de 11 de enero, sobre derechos y libertades de los extranjeros en España y su integración social, conocida popularmente como Ley de extranjería (España, 2000). Los medios de comunicación se han hecho eco de la Ley de extranjería desde su nacimiento, desde sus frecuentes modificaciones a los problemas derivados de su aplicación. Temas como los derechos y deberes de los extranjeros, su papel en una sociedad no acostumbrada a una presencia tan significativa de personas no autóctonas, la regularización de quienes se encuentran en situación ilegal o el control de las fronteras, entre muchos otros, han sido protagonistas de miles de artículos periodísticos en las dos últimas décadas.

Este estudio es fruto de una investigación más amplia que analiza múltiples cuestiones relacionadas con el tratamiento que ha hecho la prensa española de la Ley de extranjería durante el período 2003 a 2016. Este trabajo se va a centrar en mostrar cómo los diferentes actores sociales, en especial los partidos políticos, que se manifiestan a través de seis diarios seleccionados, usan sus discursos para hacer política al hablar de la Ley de extranjería como instrumento fundamental en la gestión de la multiculturalidad que conlleva la inmigración.

Para lograr este objetivo, en este artículo se siguen de forma especial los trabajos de Zapata-Barrero $(2008 ; 2009)$ sobre los aspectos discursivos relativos a la inmigración. Este autor considera al discurso como acción política: esto significa que la construcción de un discurso político tiene unas intenciones, busca tener un efecto determinado y tiene una orientación hacia la acción, por lo que es un acto político y se convierte en política. De ahí que lo denomine como una "política del discurso". Pero, ¿qué es lo político desde un punto de vista discursivo?

Hay que tener presente que existe un análisis lingüístico del discurso político que relaciona las particularidades del comportamiento lingüístico con la política o comportamiento político. Esto plantea, según Chilton y Schäffner (2008), dos problemas:

- lo que se considere "político" dependerá del punto de vista del comentarista: se definen como potencialmente políticas aquellas acciones (lingüísticas o no) que involucran al poder o a su opuesto, la resistencia. A esto hay que añadir que el concepto de poder tampoco está cerrado;

- los múltiples actos Ilevados a cabo mediante el lenguaje cumplen diversas funciones, no sólo políticas.

Estos autores concluyen que aquello que se puede considerar como "político", depende de los participantes, existiendo dos perspectivas:

a) textos donde se tratan ideas políticas, creencias y prácticas de una sociedad o de parte de ella (no es necesario que los productores de los textos sean sólo políticos): se trata del "discurso metapolítico";

b) textos de un grupo político o ideológico, o un partido. Hay diversos tipos de discursos en este grupo:

- intraestatal (interno o doméstico);

- interestatal (entre estados);

- discurso político interior (los políticos hablan y planifican entre ellos);

- discurso político externo (los políticos se comunican con el público).

En este trabajo se hará especial incidencia en el discurso "metapolítico", en el que participan diferentes actores sociales, pero también en el discurso más estrictamente político, externo, encarnado por las manifestaciones de los representantes de los propios partidos políticos y que aparecen reflejadas en la prensa.

Este trabajo muestra cómo los diferentes actores sociales, en especial los partidos políticos, usan sus discursos para hacer política al hablar de la Ley de extranjería

No se puede olvidar la importancia del papel de la prensa en un sistema político. Gomis (1974) distingue entre la tarea propia de los medios, que es la interpretación, y su función política, que es la mediación. Como mediador, su función es aproximar a autoridades y sociedad civil, proponiendo modos de integrar los intereses de ambos. El rol de mediador del periódico será más completo cuanto menor sea la diferencia entre el volumen de hechos provocados por el sistema político y el provocado por el ambiente social. Si el periódico se limita a trasladar al ambiente social las salidas (outputs) o productos del sistema político, ejercerá el papel de transmisor, pero no de mediador. Si es al contrario, ejercerá de mediador.

En esta misma línea, Casero-Ripollés (2012) considera que la mediación es una de las funciones normativas del periodismo en democracia, lo que otorga a los periodistas un elevado poder simbólico para construir la realidad social y política. Al realizar esta tarea, los periodistas asumen el rol de representantes de los ciudadanos ante la clase política.

Borrat (1989) hace hincapié en que el análisis del discurso periodístico y de los lenguajes políticos que en él se articulan permite rastrear en los textos importantes indicios de las decisiones tomadas por el periódico en cuanto a excluir, incluir y jerarquizar a los actores y las fuentes de la información política, cuyo análisis permitirá precisar la línea política del periódico y los objetivos permanentes y temporales que moldean sus prácticas rutinarias y sus actuaciones estratégicas. El presente artículo pretende hacer una aportación en este sentido, al describir los discursos políticos que se muestran en seis diarios en relación con la Ley de extranjería.

Existe una amplia bibliografía sobre el tratamiento de la inmigración en la prensa, entre la que podemos citar las obras de Checa y Arjona (2011), Igartua-Perosanz et al. (2007) y Lorite-García (2002), que analizan varias perspectivas del tratamiento informativo de la inmigración en casos concretos y con enfoques metodológicos diversos (agenda setting, framing), así como la influencia que tiene la prensa en la conformación de la opinión pública en relación con este tema. 
Sobre el aspecto multicultural de la inmigración y el papel de la prensa en este ámbito, son de interés las investigaciones de Bañón-Hernández (2007a; 2007b), que hacen referencia al papel de los medios como mediadores interculturales, ya que su función es determinante en el plano ideológico o simbólico, pero también como educadores en las interrelaciones sociales, papel que, con frecuencia, no realizan.

Casero-Ripollés (2007) se fija en la identidad mediática de los inmigrantes, que requiere una aproximación interdisciplinar, como la que se propone en esta investigación, deteniéndose en la función de los medios como auténticos gestores de identidades públicas, ya que a través de sus discursos, aquellos determinan quiénes son representados y qué marcas y significados se asocian a cada colectivo. Además, hace hincapié en la eficacia del uso de mecanismos discursivos para legitimar las políticas migratorias restrictivas.

En esto coinciden los trabajos de Martín-Rojo y Whittaker (1998) y Wodak y Meyer (2003). Estos últimos autores, junto con De-la-Fuente-García (2007), Delgado-Godoy (2007), Van-Dijk (2007; 2008; 2009); Van-Dijk y Zapata-Barrero (2007) representan el enfoque de los que, desde los métodos del análisis crítico del discurso, se han centrado especialmente en el estudio del discurso racista y en la relación entre el grupo endógeno (los ciudadanos, los autóctonos) y el exógeno (los extranjeros). A partir de estos trabajos se ha confirmado la importancia que la orientación interdisciplinar ha de tener en el análisis del discurso en un tema como la inmigración y la multiculturalidad, así como las posibilidades que ofrece la perspectiva histórica en dicho análisis del discurso, que permite descubrir cambios diacrónicos en ellos y que son especialmente relevantes en este artículo.

Finalmente, las investigaciones centradas en los discursos parlamentarios sobre inmigración de Bañón-Hernández y Fornieles (2008), Márquez-Lepe (2007), Zapata-Barrero (2008; 2009) y Zapata-Barrero; Van-Dijk (2007) han ofrecido un interesante punto de partida para determinar la existencia o no de coincidencias en contenidos, tópicos y categorías temáticas entre los discursos parlamentarios, mucho más extensos y elaborados, y los de la prensa, sobre todo cuando la fuente informativa principal son los mismos partidos políticos. Dicho aspecto también se analiza en este trabajo a partir del modelo que se comenta a continuación.

Para esta investigación se ha seguido el planteamiento de Zapata-Barrero (2009) de que el marco de referencia para abordar la política del discurso sobre la inmigración es el conflicto entre monoculturalidad y multiculturalidad. La política del discurso puede producir dos tipos de discursos (Zapata-Barrero, 2009, p. 58):

- el que lleva al pasado monocultural, al que se denomina reactivo porque reacciona contra el proceso histórico y busca restablecer dicho pasado;

- el que lleva al futuro multicultural, proactivo, que asume la irreversibilidad del proceso y busca conformarlo como marco para orientar los cambios sociales.

El discurso reactivo busca gestionar el conflicto y se preocupa por las alteraciones negativas que supone el proceso de multiculturalidad. Lo interpreta como algo negativo, como una amenaza. Es un conflicto de intereses entre ciudadanos e inmigrantes. Tiene en cuenta sólo a la población ciudadana, que siempre tiene prioridad sobre la no-ciudadana. A su vez, el discurso reactivo puede seguir dos tipos de retóricas: el ciudadanismo y el tradicionalismo.

\section{El discurso reactivo busca gestionar el conflicto y se preocupa por las alteracio- nes negativas que supone el proceso de multiculturalidad}

Por contra, el discurso proactivo aspira a proporcionar a las personas recursos para que gestionen los conflictos, percibidos como un hecho histórico irreversible. Se ve como una oportunidad, un reto. El marco de referencia es toda la población, sea ciudadana o no, votante o no. Los discursos proactivos tienen la función de socializar el proceso para que todas las formas de expresión cultural puedan ser objeto de los principios de la democracia, siempre que todas las partes respeten el pluralismo. Las dos expresiones retóricas de este discurso son la retórica de la igualdad y la de la ciudadanía.

Tabla 1. Ideas principales de las retóricas de los discursos re-activos y pro-activos

\begin{tabular}{|c|c|}
\hline Discurso reactivo & Discurso proactivo \\
\hline Retórica del ciudadanismo & Retórica de la ciudadanía \\
\hline $\begin{array}{l}\text { Populismo: prima el interés del ciudadano frente al del inmigrante en las } \\
\text { zonas de conflicto multiculturales. }\end{array}$ & $\begin{array}{l}\text { Inclusión: el inmigrante forma parte de un proceso que debe finalizar ne- } \\
\text { cesariamente en el reconocimiento pleno de la ciudadanía. }\end{array}$ \\
\hline $\begin{array}{l}\text { Esencialismo: los intereses y las necesidades de los ciudadanos son vistos } \\
\text { como inmutables, como los únicos criterios para construir un discurso. }\end{array}$ & $\begin{array}{l}\text { Acomodación: la categoría del inmigrante debe ir acompañada de la del } \\
\text { ciudadano, buscando argumentos para su adaptación mutua. }\end{array}$ \\
\hline Retórica del tradicionalismo & Retórica de la igualdad \\
\hline $\begin{array}{l}\text { Tradición e identidad nacional: recurre al pasado para formar sus argu- } \\
\text { mentos. La inmigración conlleva un conflicto que es expresión de una } \\
\text { alteración de los valores de la tradición que sustentan la estructura de la }\end{array}$ & $\begin{array}{l}\text { Carácter normativo: expresa la necesidad de tener como criterio el prin- } \\
\text { cipio de igualdad de oportunidades para gestionar las zonas de conflicto } \\
\text { multiculturales. No significa igualdad como idéntico, sino como diferencia. }\end{array}$ \\
\hline sociedad. Defensor de la identidad nacional. & Imparcialidad: no se privilegia una cultura determinada al gestionar la \\
\hline $\begin{array}{l}\text { Concepto de autoridad: con el proceso de multiculturalidad se ponen en } \\
\text { duda las estructuras de autoridad que sustentan la sociedad y las institu- } \\
\text { ciones tradicionales, lo que no es deseable. }\end{array}$ & $\begin{array}{l}\text { diferencia cultural. La idea básica es que la diferencia cultural no produz- } \\
\text { ca o consolide desventajas por razones que no dependan de la voluntad } \\
\text { de la persona. }\end{array}$ \\
\hline
\end{tabular}

Fuente: Elaborado a partir de Zapata-Barrero (2009) 
En la tabla 1 se muestran someramente las ideas principales que sustentan las retóricas que conforman los discursos reactivos y proactivos, respectivamente.

\section{Fuentes y metodología}

Las fuentes empleadas para la realización de esta investigación son seis diarios: El país, El mundo, $A B C$, Heraldo de Aragón, El periódico de Aragón y La vanguardia. Se han analizado las piezas que incluyen conjuntamente los términos "ley" y "extranjería" de todas las secciones de los diarios, incluidos todos los géneros. El período estudiado es de 14 años, para dotar de perspectiva al análisis: entre el 1 de enero de 2003 y el 31 de diciembre de 2016. Se han elegido estos medios porque incluyen tres periódicos nacionales de referencia, con líneas editoriales diferenciadas, así como otros tres diarios regionales, dos aragoneses y uno catalán. La inclusión de estos últimos pretende incorporar al estudio sensibilidades y enfoques distintos a los que aportan los diarios estatales. Se ha trabajado sólo con las versiones impresas de los diarios.

Fruto de esta búsqueda inicial se encontraron 3.428 piezas en total. Para hacer viable la investigación se decidió reducir el número de unidades de análisis que compondrían el corpus, para lo que se practicó la técnica estadística denominada "muestreo aleatorio estratificado", que es:

"aquel en el que los elementos de la población se dividen en clases o estratos. La muestra se toma asignando primero, mediante algún criterio, un número de miembros a cada estrato y escogiendo después los elementos que forman la muestra por muestreo aleatorio simple dentro del estrato" (Peña; Romo, 1997, p. 272).

En este caso, los estratos son los años en los que se han publicado las piezas de cada diario, lo que permite mantener el sustrato histórico vinculado a las vicisitudes de la Ley de extranjería a lo largo del tiempo. Tras la realización de esta operación estadística, el corpus definitivo ha quedado circunscrito a 793 unidades de análisis.

El reparto de unidades de análisis entre los seis medios, tras el muestreo, es el siguiente (la primera cifra corresponde al número total de piezas y la segunda, entre paréntesis, es la resultante tras el muestreo):

- El país: 757 (122);

- El mundo: 976 (157);

- ABC: 860 (139);

- Heraldo de Aragón: 171 (171);

- El periódico de Aragón: 122 (122);

- La vanguardia: 505 (82).

En el caso de Heraldo de Aragón y El periódico de Aragón se trabajó con el total de las piezas resultantes de la búsqueda inicial y no se realizó muestreo alguno, puesto que de haberlo hecho, el número de unidades resultante habría sido muy bajo, lo que habría menoscabado la fiabilidad del análisis.

En la metodología se ha optado por el análisis de contenido cuantitativo, que gira en torno a las herramientas estadísticas empleadas para obtener los datos descriptivos de una serie de variables. Para Berelson (1952, p. 18) el aná- lisis de contenido es una herramienta "para la descripción objetiva, sistemática y cuantitativa" de los textos comunicativos. Este método ofrece unos datos precisos a través de indicadores de frecuencia y de las interrelaciones existentes entre las variables evaluadas. Se centra en la frecuencia de determinados factores del contenido, a través de los cuales el investigador busca dar sentido a esta ocurrencia, co-ocurrencia de términos, conceptos o características del contenido. En nuestra investigación se incluyó un conjunto de variables que determinaba cuáles eran las fuentes principales y secundarias empleadas en cada unidad de análisis (administraciones públicas, gobiernos centrales, autonómicos o locales, partidos políticos de la oposición, ONGs, abogados o sindicatos, entre otros, hasta un total de 23 posibles fuentes). Para este estudio se entiende por fuente principal aquella que se muestra claramente como preponderante en la pieza analizada. Esta preponderancia se valora atendiendo a los siguientes criterios:

- mayor extensión de las manifestaciones de la fuente dentro del texto de la pieza en relación con las demás;

- presencia de la mención a la fuente en el título, subtítulo, antetítulo y/o entradilla de la pieza.

Se parte del modelo que Zapata-Barrero (2009) utilizó para estudiar los discursos sobre inmigración de los grupos parlamentarios del Congreso de los Diputados y establecer una serie de categorías temáticas sobre la tipología ya explicada, que se aplica en este trabajo.

Se trata de categorizar mediante las herramientas del análisis de contenido, dónde se encuadran discursivamente las unidades de análisis que han sido seleccionadas por tratar la Ley de extranjería, prestando especial atención a la influencia de los partidos políticos cuando son utilizados como fuente principal. En este caso, se ha puesto en relación la presencia en cada unidad de análisis de cada una de las cuatro retóricas posibles, dos pertenecientes al discurso reactivo (ciudadanismo y tradicionalismo) y dos al proactivo (ciudadanía e igualdad), tanto si aparecen de forma única (si sólo se encuentra un tipo de retórica en la pieza) o determinando si se produce la combinación entre dos de ellas (por ejemplo, tradicionalismo con igualdad) en una misma pieza. Se han limitado las combinaciones a dos, ya que es extremadamente raro que en una misma pieza periodística, dada su extensión, se puedan encontrar más retóricas presentes.

También se ha incluido la variable "ningún discurso o no se identifica con claridad" para los casos en los que no se pudiera etiquetar la pieza con alguna de las variables discursivas. La forma de operacionalizar la identificación de las retóricas presentes en cada unidad ha sido analizar cada texto y determinar la presencia o no de las retóricas presentes en él, codificándolas según el planteamiento explicado. En total hay once variables previstas, como se muestra en la tabla 2.

Las preguntas que se responderán en este trabajo son:

a) ¿qué tipo de discursos (proactivos, reactivos o mixtos) prevalecen en cada uno de los medios analizados?

b) ¿qué influencia tienen los partidos políticos en la presencia de uno u otro discurso? 


\section{Análisis de resultados}

\subsection{Discursos y retóricas sobre la Ley de extranjería}

A partir del planteamiento metodológico expuesto, tras codificar todas las unidades de análisis según su adscripción a una u otra retórica discursiva [tradicionalismo y ciudadanismo dentro de los discursos reactivos $(R)$, igualdad y ciudadanía dentro de los proactivos $(P)$ o combinación de retóricas reactivas y proactivas para crear discursos mixtos (M)], la tabla 2 muestra en porcentaje dentro de cada diario cómo se reparten las piezas analizadas según su pertenencia a una u otra retórica. También se proporcionan datos para el conjunto de los diarios seleccionados en las dos columnas de la derecha.

Del total de 793 piezas que componen el corpus, 277 (34,9\%) presentan una retórica de igualdad, una de las dos de tipo proactivo. Le sigue la retórica reactiva del tradicionalismo, con un $24,6 \%$ de las unidades en el conjunto de los periódicos. Son las dos retóricas predominantes:

- una es proclive a otorgar a los inmigrantes las mismas oportunidades que a los ciudadanos autóctonos;

- la otra expresa la resistencia al cambio, desde el principio de la autoridad y el inmovilismo.

La tercera retórica por peso relativo es la reactiva del ciudadanismo $(9,6 \%)$, de tipo populista, y que tiene presentes en primer lugar a los ciudadanos españoles como sus referentes principales, a los que debe atender de forma prioritaria. El resto de combinaciones mixtas, tanto dentro de un mismo discurso, como mezclando tipos de discurso proactivos y reactivos, tienen una presencia poco relevante.
Finalmente se han encontrado 103 unidades (13\%) donde no ha sido posible categorizar el discurso presente siguiendo el modelo planteado.

Si agrupamos las retóricas en sus respectivos tipos discursivos, la imagen se aclara bastante. El gráfico 1 permite observar cuáles son los discursos presentes en cada uno de los seis diarios analizados y en su conjunto.

Considerando los datos del conjunto de los medios, en la columna derecha del gráfico se observa que el $36,9 \%$ de las unidades presentan un discurso reactivo, frente a un $39,6 \%$ proactivo. Hay un $10,5 \%$ de unidades que combinan ambos y un $13 \%$ donde no ha sido posible identificar ninguno. Existe por tanto bastante igualdad en el conjunto de los medios a la hora de mostrar ambos discursos.

\section{El discurso proactivo aspira a proporcio-} nar a las personas recursos para que gestionen los conflictos, percibidos como un hecho histórico irreversible

Sin embargo, el panorama cambia cuando nos fijamos en cada uno de los medios. Así, El país está por debajo del dato conjunto en sus discursos reactivos, ya que suponen un $31,1 \%$ de sus piezas, pero también en los proactivos $(36,9 \%$ frente a $39,6 \%)$. Destaca la retórica de la igualdad (31,1\%). La diferencia entre discursos proactivos y reactivos es de casi seis puntos a favor de los primeros. Este medio incluye discursos mixtos en una misma pieza en una proporción mucho mayor que los demás (17,2\% frente al dato global de $10,5 \%$ ).

Tabla 2. Retóricas discursivas por diarios y en conjunto

\begin{tabular}{|c|c|c|c|c|c|c|c|c|}
\hline & Elpaís & El mundo & $A B C$ & $\begin{array}{c}\text { Heraldo de } \\
\text { Aragón }\end{array}$ & $\begin{array}{c}\text { El periódico } \\
\text { de Aragón }\end{array}$ & $\begin{array}{c}\text { La } \\
\text { vanguardia }\end{array}$ & \multicolumn{2}{|c|}{ Conjunto de diarios } \\
\hline & \multicolumn{6}{|c|}{$\%$ dentro del diario } & $\begin{array}{c}\mathrm{N}^{\circ} \text { de } \\
\text { unidades }\end{array}$ & $\%$ \\
\hline Tradicionalismo (R) & 13,1 & 29,9 & 44,6 & 20,5 & 10,7 & 26,8 & 195 & 24,6 \\
\hline Ciudadanismo (R) & 14,8 & 11,5 & 8,6 & 7,6 & 3,3 & 13,4 & 76 & 9,6 \\
\hline lgualdad (P) & 31,1 & 31,2 & 12,9 & 45,6 & 52,5 & 36,6 & 277 & 34,9 \\
\hline Ciudadanía (P) & 3,3 & 1,9 & 2,2 & 4,1 & 5,7 & 1,2 & 25 & 3,2 \\
\hline $\begin{array}{l}\text { Tradicionalismo y } \\
\text { ciudadanismo (R) }\end{array}$ & 3,3 & 4,5 & 5,0 & 0,6 & 0,8 & 2,4 & 22 & 2,8 \\
\hline $\begin{array}{l}\text { Igualdad y } \\
\text { ciudadanía (P) }\end{array}$ & 2,5 & 0,6 & 0,0 & 2,3 & 1,6 & 2,4 & 12 & 1,5 \\
\hline $\begin{array}{l}\text { Tradicionalismo e } \\
\text { igualdad (M) }\end{array}$ & 6,6 & 6,4 & 5,0 & 3,5 & 1,6 & 3,7 & 36 & 4,5 \\
\hline $\begin{array}{l}\text { Tradicionalismo y } \\
\text { ciudadanía (M) }\end{array}$ & 0,0 & 0,6 & 0,0 & 0,0 & 0,0 & 0,0 & 1 & 0,1 \\
\hline $\begin{array}{l}\text { Ciudadanismo e } \\
\text { igualdad (M) }\end{array}$ & 8,2 & 6,4 & 4,3 & 3,5 & 3,3 & 6,1 & 41 & 5,2 \\
\hline $\begin{array}{l}\text { Ciudadanismo y } \\
\text { ciudadanía (M) }\end{array}$ & 2,5 & 0,0 & 0,0 & 0,0 & 1,6 & 0,0 & 5 & 0,6 \\
\hline $\begin{array}{l}\text { Ninguno o no se } \\
\text { identifica }\end{array}$ & 14,8 & 7,0 & 17,3 & 12,3 & 18,9 & 7,3 & 103 & 13,0 \\
\hline $\begin{array}{l}\text { Total (\% y número de } \\
\text { unidades) }\end{array}$ & $100(122)$ & $100(157)$ & 100 (139) & $100(171)$ & $100(122)$ & $100(82)$ & 793 & 100 \\
\hline
\end{tabular}




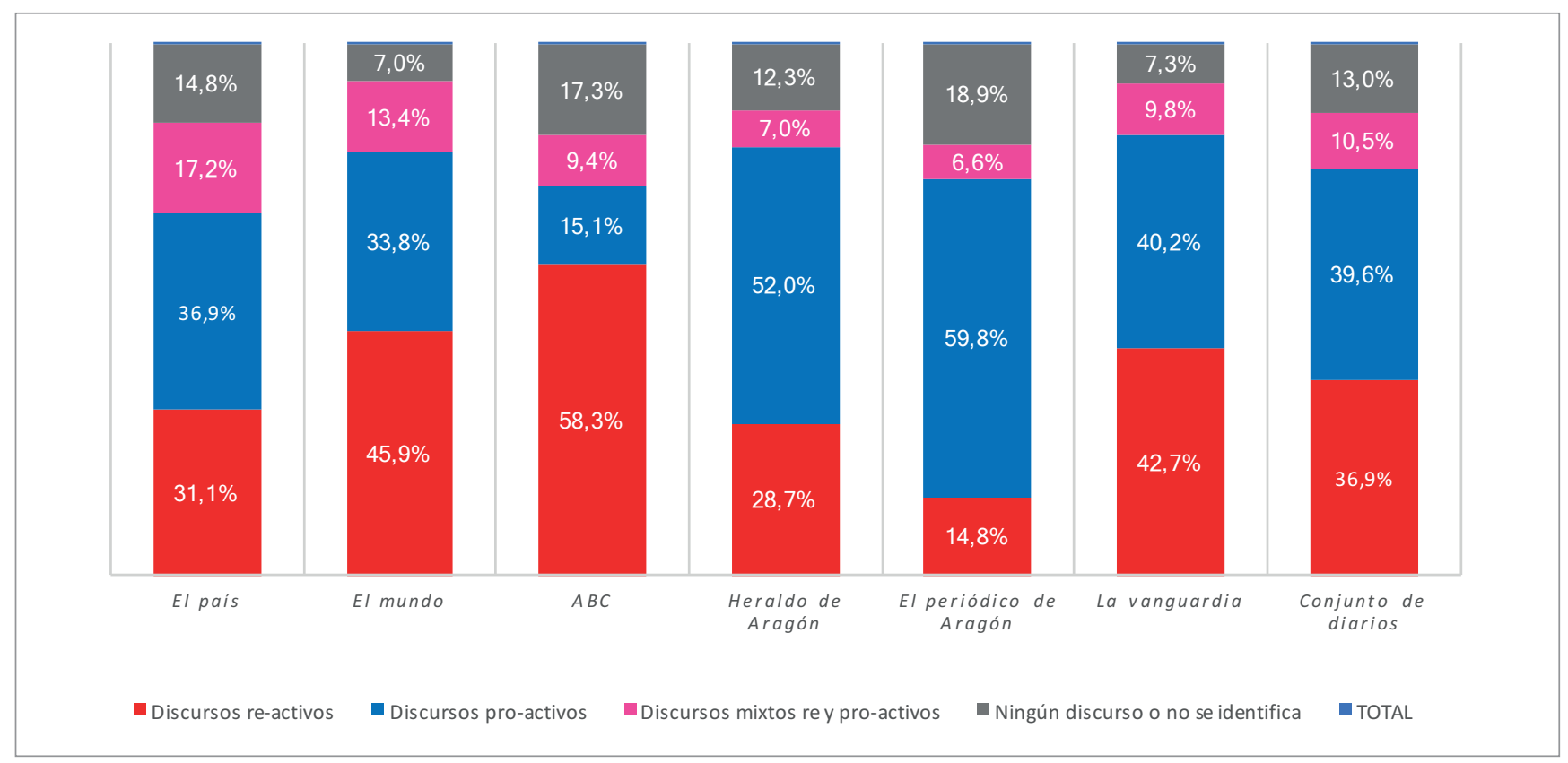

Gráfico 1. Tipos de discursos por diarios y en conjunto (\%)

El mundo se caracteriza por la importancia de la retórica tradicionalista (29,9\%, frente al dato conjunto de $24,6 \%$ ), sólo por detrás de $A B C(44,6 \%)$. El resto de sus piezas se encuentran muy cercanas al dato del conjunto de diarios. Además, es donde con mayor claridad se pueden categorizar dentro de un tipo de retórica u otra, ya que sólo el 7\% de las mismas no se ha podido incluir en alguna de ellas. Si nos fijamos en los datos agrupados, los discursos reactivos (45,9\%, cuando en el conjunto es de 36,9\%) prevalecen claramente sobre los proactivos (33,8\%, dato global de $39,6 \%$ ), con una diferencia de 12 puntos.

$A B C$ tiene muy claro su enfoque: predomina la retórica tradicionalista con un $44,6 \%$ de sus piezas, doblando casi el resultado del conjunto de diarios (24,6\%). Además es el diario que menos veces ofrece una retórica de igualdad (sólo el $12,9 \%$ de las veces, cuando el dato global es del 34,9\%). Si acudimos a los datos agrupados, es significativo que el $58,3 \%$ de las piezas de $A B C$ presentan discursos reactivos, mientras los proactivos suponen un $15,1 \%$. La diferencia entre ambas es de 43 puntos porcentuales.

\section{Es destacable la igualdad casi absoluta entre discursos proactivos y reactivos en el conjunto de los medios analizados, si bien cada diario tiene sus características propias}

Pasando a los diarios regionales, Heraldo de Aragón se inclina por la retórica de la igualdad $(45,6 \%$, el segundo que más lo hace). Con los datos agrupados, los discursos proactivos suman la mitad de las piezas (52\%), mientras los reactivos son un $28,7 \%$ del total. La diferencia entre ambos es de 23 puntos a favor de los proactivos. También es el segundo medio que menos mezcla ambos tipos de discurso, sólo el $7 \%$ de las ocasiones.
El otro diario aragonés, El periódico de Aragón, también es diáfano: el $52,5 \%$ de sus piezas abogan por la igualdad. Abundando en el discurso proactivo, es el que más incide en la retórica de la ciudadanía $(5,7 \%$, cuando el dato global es del $3,2 \%)$. Por el contrario, el $18,9 \%$ de sus piezas no presentan un discurso claro, siendo el diario al que más le ocurre, por encima de $A B C$. Si nos fijamos en los datos agrupados, El periódico de Aragón es el diario que presenta de forma más evidente su predilección por los discursos proactivos (un $59,8 \%$ de sus piezas) y su fobia por los reactivos (14,8\%, con muchísima diferencia sobre el dato global de $36,9 \%$ ). La diferencia en puntos porcentuales entre ambas es de 45 puntos a favor de los discursos proactivos, lo que convertiría a este medio en una especie de antítesis de $A B C$, que se coloca en la posición totalmente opuesta, con casi la misma diferencia en puntos porcentuales. También es el que menos mezcla discursos proactivos y reactivos, sólo el $6,6 \%$ de las veces.

Finalmente, La vanguardia se encuentra en torno a los datos del conjunto de diarios, al prevalecer la retórica de la igualdad, con el $36,6 \%$ (frente al dato global del 34,9\%), con el contrapeso de la tradicionalista $(26,8 \%$, algo por encima del $24,6 \%$ de todos los medios conjuntamente). Junto con El país es el que menos piezas sin categorizar tiene (7,3\%). Agrupando las retóricas y los discursos, se trata de un periódico que guarda equilibrio entre discursos reactivos $(42,7 \%)$ y proactivos, que quedan algo por debajo $(40,2 \%)$, si bien los primeros están por encima del dato global.

\subsection{La retórica dominante en función de la fuente po- lítica principal}

Hasta este momento se han mostrado los discursos presentes en las unidades de análisis, sin hacer distinción entre sus emisores. Para poder atribuirles discursos concretos, lo primero es determinar las fuentes que los emiten y los medios que los presentan. 
Dados los límites de este artículo, en este epígrafe se analizan sólo los datos relacionados con las fuentes más puramente políticas, vinculadas a gobiernos y administraciones, así como a partidos políticos. Hay que señalar que, de todas las fuentes que aparecían como principales en el corpus, el $40 \%$ eran puramente políticas, lo que denota su importancia. Cada una de estas fuentes pertenece a un determinado partido político, que es otra variable que se tuvo en cuenta y que permite dotar de color político a los diferentes discursos.

De todos los partidos, los cinco que aparecen como fuente principal con mayor frecuencia son:

- Partido Socialista Obrero Español/Partido de los Socialistas de Cataluña: PSOE/PSC (155 unidades; $51,5 \%$ del total comparado con el resto de partidos);

- Partido Popular: PP (109; 36,2\%);

- Convergència i Unió: CiU (12; 4\%);

- Chunta Aragonesista: CHA (8; 2,7\%);

- Izquierda Unida: IU (7; 2,3\%).

La tabla 3 muestra las retóricas de cada uno de los partidos políticos cuando son la fuente principal de la unidad analizada. Cuando el PSOE/PSC es la fuente principal, se encuentra la retórica proactiva de la igualdad $(29,7 \%)$ en la pieza periodística en que se inserta, seguida por las reactivas del tradicionalismo $(18,7 \%$ ) y el ciudadanismo $(12,9 \%)$. Aquellas piezas que no permiten identificar ninguna retórica cuando la fuente principal es el PSOE/PSC suman el $16,8 \%$ dentro de la tipología de este partido.

Cuando es el $P P$ la fuente principal, las retóricas tienden claramente hacia las retóricas reactivas del ciudadanismo $(25,7 \%)$ y el tradicionalismo $(25,7 \%)$. Las piezas mixtas que unen ciudadanismo e igualdad son un $13,8 \%$, igual que las que no permiten identificar ningún discurso multicultural $(13,8 \%)$.

La tercera fuerza política que se presenta alguna vez como fuente principal es $\mathrm{CiU}$, con sólo 12 apariciones, seguida de CHA con 8 e IU con 7. La presencia de CHA se explica por dos razones:

- selección de dos medios aragoneses;

- existencia de diputados de esta formación en el Congreso de los Dipytados en buena parte del período elegido.

Al observar los datos agrupados por discursos proactivos o reactivos (gráfico 2), cuando el PSOE es la fuente principal, las piezas en que aparece ofrecen un discurso casi equidistante entre lo reactivo ( $32,3 \%$ de las piezas) y lo proactivo $(35,5 \%)$, teniendo un $15,5 \%$ de las unidades de análisis un contenido mixto. Si es el $P P$ la fuente principal de la pieza, el discurso aparece mucho más sesgado: el $57,8 \%$ de aquellas promueven discursos reactivos, y sólo el 6,4\% proactivos, quedando un $22 \%$ para los discursos mixtos.

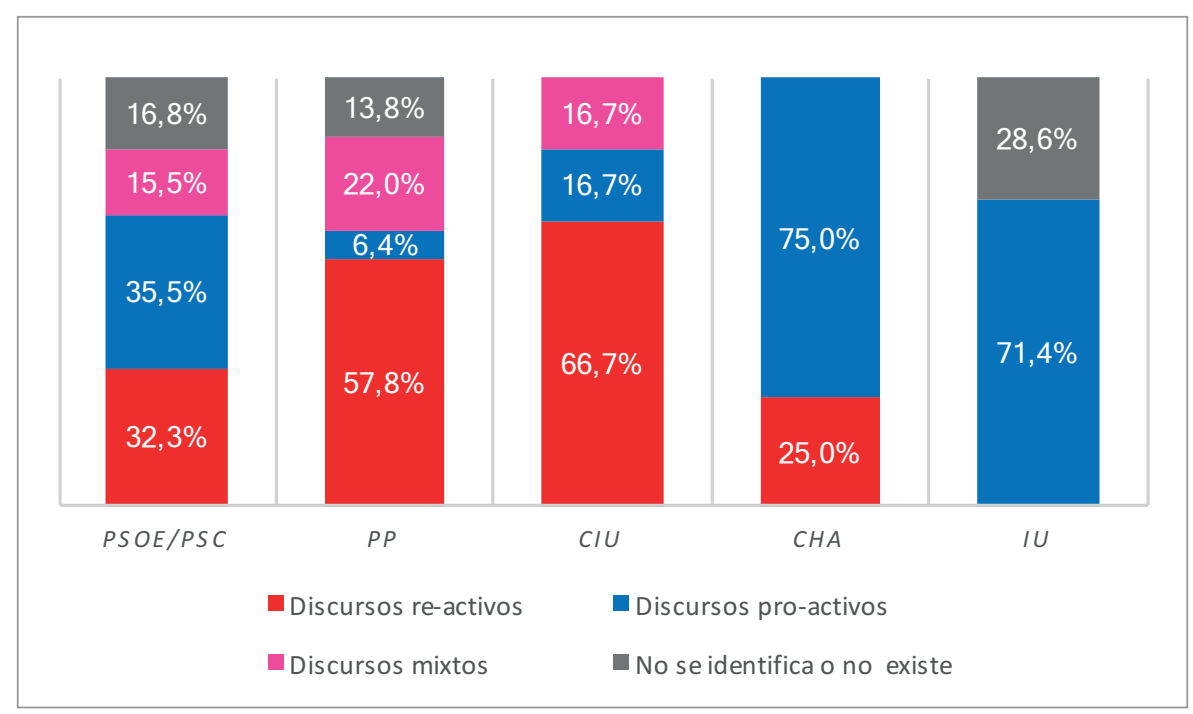

Gráfico 2. Tipos de discursos por partidos políticos cuando son fuente principal (\%)
Ahondando en el análisis, se ha querido observar si la presencia en el Gobierno de PSOE y PP ha alterado de algún modo su discurso político tal como aparece en la prensa. Comenzando por el $P S O E$, sus dos etapas de gobierno (VIII y IX Legislaturas) tienen la particularidad de que son dos los ministros responsables de inmigración en cada una de estas legislaturas que además tuvieron a priori planteamientos y talantes diversos en relación con la extranjería. Se trata de los ministros Jesús Caldera (2004-2008) y José Corbacho (2008-2011). Por otra parte, en 2008 coincidiendo con el segundo gobierno so- 
cialista, se hizo patente la crisis económica en España y parece oportuno observar si hubo cambios en el discurso sobre el papel de la Ley de extranjería en esta coyuntura. En la tabla 4 se han incluido los datos relativos al período en el que gobernó el PSOE (02/04/2004 - 20/11/2011) y también para cada uno de los dos gobiernos socialistas por separado, para poder apreciar las diferencias, en este caso ya agrupadas por discursos proactivos y reactivos o mixtos.

De estos datos se desprende que durante el primer gobierno del PSOE predominan los discursos proactivos (44,8\%) sobre los reactivos, que siguen siendo relevantes $(27,1 \%)$. En el segundo gobierno socialista, la balanza se mueve hacia los discursos reactivos $(43,8 \%)$, quedando los proactivos $(18,8 \%)$ muy devaluados. Recordemos que el dato de todo el período de gobierno socialista es de $32,6 \%$ para discursos reactivos y $36,1 \%$ proactivos. También se duplica el peso de los discursos mixtos en el segundo período socialista $(20,8 \%)$ frente al $11,5 \%$ del primer gobierno (el dato global es $14,6 \%)$.

Al observar los datos relativos al $P P$ cuando es fuente principal en las dos etapas de gobierno (01/01/2003-01/04/2004 y $21 / 11 / 2011-31 / 12 / 2016)$, hay que tener en cuenta que en el primer período, gobernando José María Aznar, el peso de los discursos reactivos $(63,9 \%)$ es ligeramente superior al del conjunto del período $(59,2 \%)$, frente al tiempo de presidencia del gobierno de Mariano Rajoy, donde descienden algo hasta el 55,9\%. En cuanto a los proactivos, dentro de su escasísima representación, son más visibles en el primer período ( $8,3 \%)$, que en el segundo $(2,9 \%)$, siendo el dato de conjunto de 5,6\%. En los discursos mixtos es donde se aprecian mayores diferencias, destacando que las piezas de este tipo en el período de Rajoy son un $26,5 \%$, cuando el dato del conjunto del período de gobierno popular es de $19,7 \%$. En definitiva, en el caso del $P P$ no hay cambios de posicionamiento llamativos entre sus dos etapas de gobierno, siendo el discurso de las piezas en las que este partido aparece como fuente principal mayoritariamente reactivo y casi nunca proactivo.

\section{Conclusiones}

Es destacable como primera conclusión la igualdad casi absoluta entre discursos proactivos y reactivos en el conjunto de los medios analizados, lo que manifiesta una visión global de los medios, dividida y contradictoria, pero sin duda reflejo de los posicionamientos de los actores sociales presentes en la prensa y por ende de nuestra sociedad, sobre cómo abordar el reto de la multiculturalidad a partir de la herramienta jurídico-política que proporciona la Ley de extranjería.

Cuando el $P P$ es la fuente principal en una pieza periodística, predomina claramente el discurso de tipo reactivo

La segunda conclusión es que los discursos mixtos, que mezclan diferentes retóricas discursivas del proceso multicultural, son ciertamente escasos (un 10,5\%), lo que manifiesta cierta homogeneidad a la hora de construir las piezas desde el punto de vista discursivo: o son reactivas o son proactivas mayoritariamente, aunque existan matices en cuanto a la retórica utilizada dentro de cada tipo de discurso. Cabe preguntarse si la calidad informativa de los medios queda en entredicho por este hecho. Si la función política de los medios es mediadora, quizá sería adecuado que existiera en cada pieza una mayor diversidad de posicionamientos que permitiera valorar diferentes perspectivas de un mismo fenómeno (la Ley de extranjería en este caso), si no en todas ellas porque no sería factible, sí en una mayor proporción de las mismas.
Tabla 5. Tipo de discursos del $P P$ cuando es fuente principal durante las etapas de gobierno de Aznar (2003-2004) y Rajoy (2011-2016) (\%)

\begin{tabular}{|l|c|c|c|}
\hline \multicolumn{1}{|c|}{$\boldsymbol{P P}$ como fuente principal } & $\begin{array}{c}\text { 1 } \text { Gobierno PP (VII } \\
\text { Legislatura: 2003- } \\
\text { 2004 - Aznar) }\end{array}$ & $\begin{array}{c}\text { 20 Gobierno PP (X } \\
\text { a XII Legis/aturas: } \\
\mathbf{2 0 1 1 - 2 0 1 6} \text { - Rajoy) }\end{array}$ & $\begin{array}{c}\text { Durante todo el pe- } \\
\text { ríodo en que gobier- } \\
\text { na el PP (2003-2004 y } \\
\mathbf{2 0 1 1 - 2 0 1 6 )}\end{array}$ \\
\hline Discursos reactivos & 63,9 & 55,9 & 59,2 \\
\hline Discursos proactivos & 8,3 & 2,9 & 5,6 \\
\hline Discursos mixtos & 11,1 & 26,5 & 19,7 \\
\hline Ningún discurso o no se identifica & 16,7 & 14,7 & 15,5 \\
\hline
\end{tabular}

De los medios analizados, dos están claramente separados de los datos del conjunto al contar con mayor presencia de los discursos reactivos: $A B C \mathrm{y}$, en menor medida, El mundo. La vanguardia está en un punto de equilibrio, mientras El país y Heraldo de Aragón están notoriamente por 
debajo, quedando claro que El periódico de Aragón apenas acepta este tipo de discurso reactivo entre sus contenidos.

Los diarios aragoneses prefieren los discursos proactivos, sin ninguna duda. El resto de medios los presentan sin dificultades, en una proporción algo más alta que los reactivos, en el caso de El país, y bastante más baja en el de El mundo, salvo $A B C$, que los ignora de forma habitual, dada la escasa aceptación entre sus piezas.

Cuando el PSOE es la fuente principal de la noticia, se mantiene un equilibrio entre los discursos proactivo y reactivo

En los discursos mixtos sólo destaca El país como el que más los entremezcla dentro de una misma pieza. En las diferencias entre diarios nacionales y regionales, se ha observado una mayor preferencia de los diarios aragoneses por los discursos proactivos frente a la escasísima presencia de los reactivos, a pesar de que las líneas ideológicas de Heraldo de Aragón y El periódico de Aragón son muy diferentes, lo que hace pensar en que dicha preferencia tiene que ver con otras consideraciones como la cercanía del medio a sus lectores o el tipo de fuentes empleadas. El caso de La Vanguardia es diferente, ya que se asemeja más a un diario nacional.

En cuanto a la segunda pregunta de investigación propuesta, sobre la influencia de los partidos políticos en la presencia de un tipo de discurso u otro en la prensa, se ha observado que en relación con los dos partidos políticos principales de nuestro país, su influencia queda demostrada: cuando el $P P$ es la fuente principal en una pieza, predomina claramente el discurso de tipo reactivo, repartido a partes iguales entre el populismo ciudadanista y el tradicionalismo conservador. El discurso proactivo apenas entra en sus parámetros. Dichas posiciones no varían excesivamente entre los gobiernos de Aznar y Rajoy. Por su parte, cuando es el PSOE la fuente principal se mantiene un equilibrio entre ambos discursos, si bien se ha observado que esta posición ha mutado entre un período más proactivo (2004-2008) y otro reactivo (2008-2011).

\section{Bibliografía}

Bañón-Hernández, Antonio-Miguel (2007a). Discurso periodístico y procesos migratorios. San Sebastián: Tercera Prensa. ISBN: 9788487303623

Bañón-Hernández, Antonio-Miguel (2007b). “Los medios como mediadores interculturales". En: Bañón-Hernández, Antonio-Miguel. Discurso periodístico y procesos migratorios. San Sebastián: Tercera Prensa, pp. 7-26. ISBN: 97884 87303623

Bañón-Hernández, Antonio-Miguel; Fornieles-Alcaraz, Javier (coords.) (2008). Manual sobre comunicación e inmigración. San Sebastián: Tercera Prensa. ISBN: 9788496993020

Berelson, Bernard (1952). Content analysis in communication research. New York: Free Press. ISBN: 0028412109 http://www.mpi.nl/publications/escidoc-2281462
Borrat, Héctor (1989). "El periódico, actor del sistema político". Anàlisi, n. 12, pp. 67-80.

https://goo.gl/rxPpXB

Casero-Ripollés, Andreu (2007). "Discurso mediático, inmigración e ilegalidad: legitimar la exclusión a través de la noticia". En: Zapata-Barrero, Ricard; Van-Dijk, Teun A. Discursos sobre la inmigración en España. Los medios de comunicación, los parlamentos y las administraciones. Barcelona: Fundación Cidob, pp. 69-90. ISBN: 9788487072789

Casero-Ripollés, Andreu (ed.) (2012). Periodismo político en España: concepciones, tensiones y elecciones. La Laguna (Tenerife): Sociedad Latina de Comunicación Social. ISBN: 978 8415698098

http://repositori.uji.es/xmlui/bitstream/handle/10234/73587/ CAL33Casero.pdf?sequence $=6$

Checa, Juan-Carlos; Arjona, Ángeles (2011). “Españoles ante la inmigración: el papel de los medios de comunicación". Comunicar, v. XIX, n. 37, pp. 141-149.

https://doi.org/10.3916/C37-2011-03-06

Chilton, Paul; Schäffner, Christina (2008). “Discurso y política". En: Van-Dijk, Teun-A. El discurso como interacción social. Estudios sobre el discurso II. Una introducción multidisciplinaria. Barcelona: Gedisa, pp. 297-330. ISBN: $847432713 X$

De-la-Fuente-García, Mario (2007). “Argumentación e inmigración. Reflexiones sobre algunas estrategias empleadas en los medios de comunicación". En: Bañón-Hernández, Antonio-Miguel. Discurso periodístico y procesos migratorios. San Sebastián: Tercera Prensa, pp. 343-374. ISBN: 97884 87303623

Delgado-Godoy, Leticia (2007). "Argumentación gubernamental y política de inmigración". En: Zapata-Barrero, Ricard; Van-Dijk, Teun A. Discursos sobre la inmigración en España. Los medios de comunicación, los parlamentos y las administraciones. Barcelona: Fundación Cidob, pp. 201-221. ISBN: 9788487072789

España (2000). “Ley orgánica 4/2000, de 11 de enero, sobre derechos y libertades de los extranjeros en España y su integración social". BOE, n. 10, 12 de enero.

https://www.boe.es/buscar/act.php?id=BOE-A-2000-544

Gomis, Lorenzo (1974). El medio media: la función política de la prensa. Madrid: Seminarios y Ediciones. ISBN: 84 29900659

Igartua-Perosanz, Juan-José; Muñiz-Muriel, Carlos; Otero-Parra, José-Antonio; De-la-Fuente-Juan, Montse (2007). "El tratamiento informativo de la inmigración en los medios de comunicación españoles. Un análisis de contenido desde la Teoría del Framing". Estudios sobre el mensaje periodístico, pp. 91-110.

http://revistas.ucm.es/index.php/ESMP/article/view/ ESMP0707110091A

Lorite-García, Nicolás (2002). Tratamiento informativo de la inmigración en España. 2002. Madrid: Ministerio de Trabajo y Asuntos Sociales. ISBN: 9788484460633

Márquez-Lepe, Esther (2007). "La gestión parlamentaria del discurso político sobre inmigración en España". En: Za- 
pata-Barrero, Ricard; Van-Dijk, Teun A. Discursos sobre la inmigración en España. Los medios de comunicación, los parlamentos y las administraciones. Barcelona: Fundación Cidob, pp. 93-127. ISBN: 9788487072789

Martín-Rojo, Luisa; Whittaker, Rachel (coords.) (1998). Poder-decir o el poder de los discursos. Madrid: Ediciones Universidad Autónoma de Madrid. ISBN: 8492379219

Peña, Daniel; Romo, Juan (1997). Introducción a la estadística para las ciencias sociales. Madrid: McGraw-Hill Interamericana de España. ISBN: 8448116178

Van-Dijk, Teun A. (2007). "El racismo y la prensa en España". En: Bañón-Hernández, Antonio-Miguel. Discurso periodístico y procesos migratorios. San Sebastián: Tercera Prensa, pp. 27-80. ISBN: 9788487303623

Van-Dijk, Teun A. (2008). "Reproducir el racismo: el rol de la prensa". En: Checa-Olmos, Francisco (coord.). La inmigración sale a la calle: Comunicación y discursos políticos sobre el fenómeno migratorio. Barcelona: Icaria. ISBN: 978 8474262490
Van-Dijk, Teun A. (2009). Discurso y poder. Barcelona: Gedisa. ISBN: 8497842820

Van-Dijk, Teun A.; Zapata-Barrero, Ricard (2007). "Introducción: inmigración y discurso". En: Zapata-Barrero, Ricard; Van-Dijk, Teun A. Discursos sobre la inmigración en España. Los medios de comunicación, los parlamentos y las administraciones. Barcelona: Fundación Cidob, pp. 9-14. ISBN: 978 8487072789

Wodak, Ruth; Meyer, Michael (2003). Métodos de análisis crítico del discurso. Barcelona: Gedisa. ISBN: 8474329701

Zapata-Barrero, Ricard (2008). Multiculturalidad e inmigración. Madrid: Síntesis. ISBN: 8497562216

Zapata-Barrero, Ricard (2009). Fundamentos de los discursos políticos en torno a la inmigración. Madrid: Trotta. ISBN: 9788498790214

Zapata-Barrero, Ricard; Van-Dijk, Teun-A. (2007). Discursos sobre la inmigración en España. Los medios de comunicación, los parlamentos y las administraciones. Barcelona: Fundación Cidob. ISBN: 9788487072789

\section{Colección EPI Scholar Libros científicos de Información, Documentación y Comunicación}

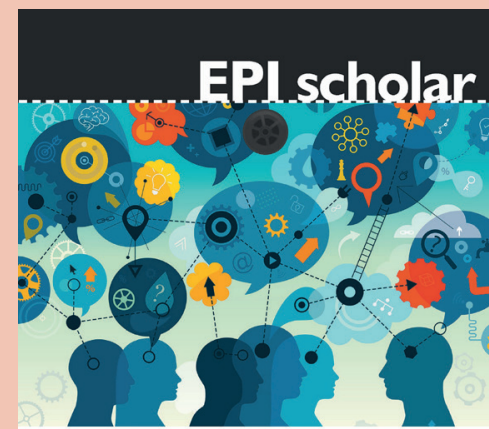

DESAFÍO A LA INVESTIGACIÓN ESTÁNDAR EN COMUNICACIÓN CRÍTICA Y ALTERNATIVAS

MANUEL GOYANES

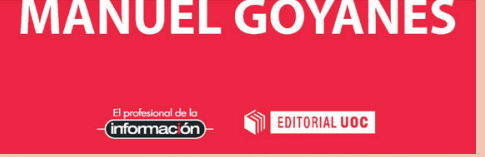

\section{Desafío a la investigación estándar en comunicación. Crítica y alternativas de Manuel Goyanes}

La investigación estándar es la visión legítima y comúnmente aceptada de lo que se considera científico. Es, a grandes rasgos, la buena ciencia. Un tipo de ciencia aplaudida por todos y de la que (casi) todos recogemos sus frutos: permite incrementar la productividad, adquirir estatus y, finalmente, notoriedad y acreditaciones. La investigación estándar es algo natural y racional, aunque también el principal factor de deshidratación intelectual de nuestro campo: inunda la investigación con formas y expresiones formularias, silencia la pluralidad de aproximaciones y fomenta el especialismo hasta lo absurdo.

Este libro aborda frontalmente la problemática de la estandarización y sus implicaciones científico-sociales. A través de su lectura, el lector descubrirá cuáles son las normas y valores que regulan la producción de conocimiento, qué hay detrás de la tierra incógnita de nuestras prácticas y disposiciones científicas y cómo desarrollar un tipo de investigación más imaginativa y original. A lo largo de sus páginas muestra cómo es posible pensar e investigar de modo alternativo a través de la problematización de lo dado por sentado, el estilo narrativo y la provocación. El libro es una llamada de atención para salir de nuestro estado de confort actual. Anima a los investigadores a retar lo establecido y a alcanzar notoriedad e impacto mediante ideas y teorías desafiantes

Goyanes, Manuel (2017). Desafío a la investigación estándar en comunicación. Crítica y alternativas. Barcelona: El profesional de la información, Editorial UOC, colección EPI Scholar n. 7, 164 pp. ISBN: 97884 91166757

Información: Isabel Olea

epi.iolea@gmail.com

http://www.elprofesionaldelainformacion.com/librosEPIScholar.html 
a $2,7,01$ मान revisers

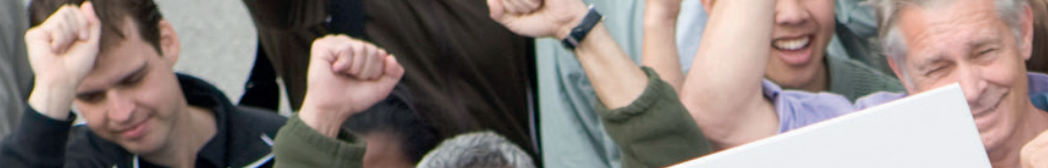

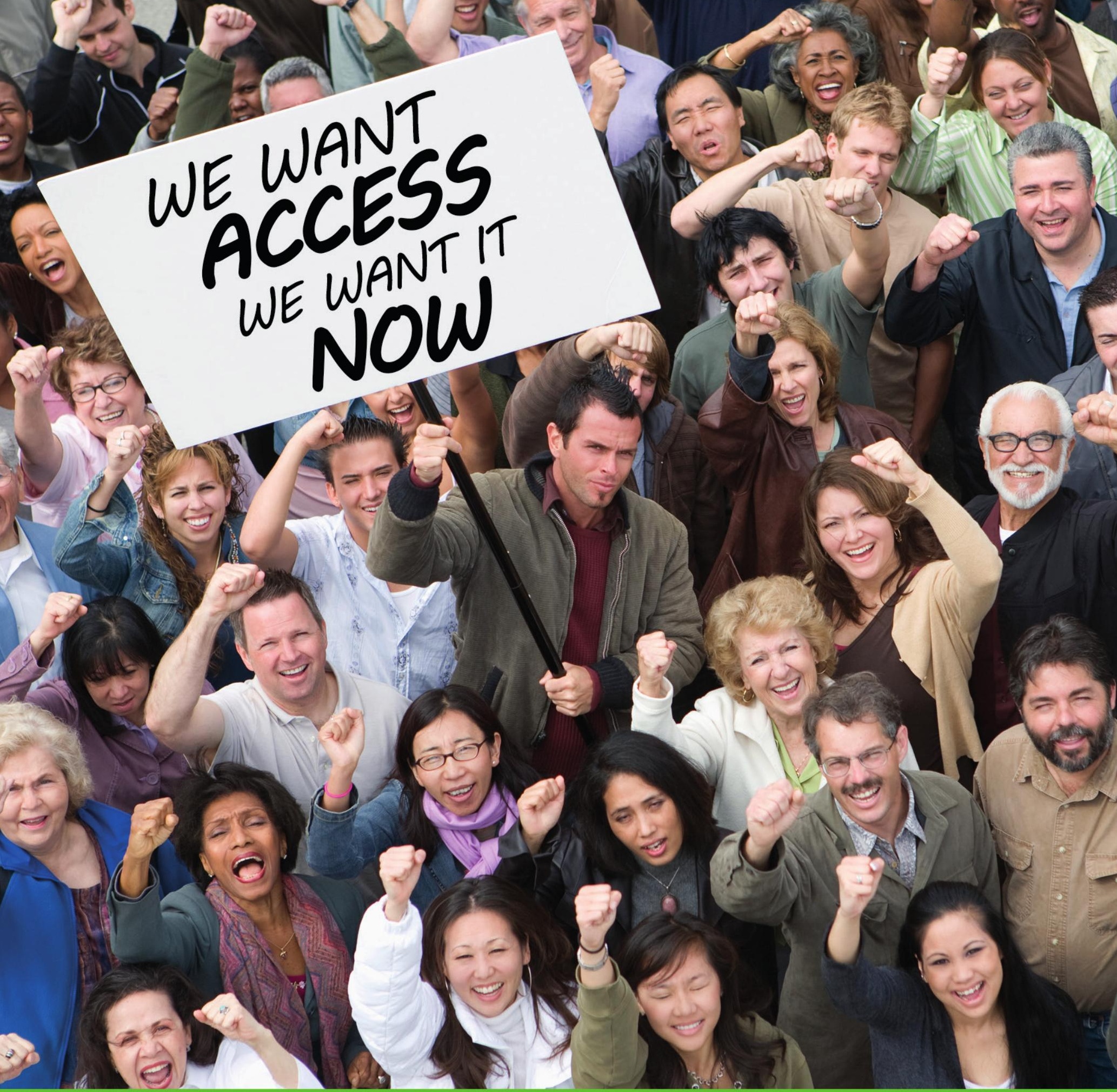

20 años diseñando

y gestionando información

MASmedios apoya la Declaracion de Lyon del 2014 que

propugna el derecho de las personas a acceder a la información. masmedios

www.masmedios.com 